\title{
Pharmacokinetic evaluation and antitumor potency of liposomal nanoparticle encapsulated cisplatin targeted to CD24-positive cells in ovarian cancer
}

\author{
KEISUKE ASHIHARA ${ }^{1}$, YOSHITO TERAI ${ }^{2}$, TOMOHITO TANAKA ${ }^{1}$, YOSHIMICHI TANAKA ${ }^{1}$, SATOE FUJIWARA ${ }^{1}$, \\ KAZUYA MAEDA $^{1}$, SATOSHI TUNETOH ${ }^{1}$, HIROSHI SASAKI ${ }^{1}$, MASAMI HAYASHI ${ }^{1}$ and MASAHIDE OHMICHI ${ }^{1}$ \\ ${ }^{1}$ Department of Obstetrics and Gynecology, Osaka Medical College, Takatsuki, Osaka 569-8686; \\ ${ }^{2}$ Department of Obstetrics and Gynecology, Kobe University Graduate School of Medicine, Kobe, Hyogo 650-0017, Japan
}

Received June 2, 2019; Accepted October 16, 2019

DOI: $10.3892 / \mathrm{ol} .2020 .11279$

\begin{abstract}
CD24, which is upregulated in several human malignancies, is related to Epithelial-mesenchymal-transition (EMT) and has characteristics of cancer stem-like cells, especially in cisplatin-resistant ovarian carcinoma cells. Drug delivery systems represent a promising therapeutic approach for diseases with treatment resistance, and the present study investigated a novel CD24-targeted drug delivery system for advanced ovarian carcinoma. We produced liposomal cisplatin with a red fluorescent substance - cyanine 5.5 (GL-CDDP-Cy5.5). In order to target CD24-positive cells, an anti-CD24 monoclonal antibody was modified to the above drug (CD24-GL-CDDP-Cy5.5). Specific uptake of CD24-GL-CDDP-Cy5.5 was confirmed using a therapeutically resistant ovarian cancer cell line, Caov-3 cells. Antitumor effects of CD24-GL-CDDP-Cy5.5 were then evaluated in Caov-3 xenograft mice. CD24-GL-CDDP-Cy5.5 showed more specific uptake by flow cytometry than GL-CDDP-Cy5.5.In xenograft mice, GL-CDDP-Cy5.5 and CD24-GL-CDDP-Cy5.5 treatment had significantly higher platinum concentration in disseminated tumor cells than cisplatin $(\mathrm{P}<0.05)$. Moreover, CD24-GL-CDDP-Cy5.5 suppressed tumor growth and prolonged survival time compared with other treatments. Median survival times of the control, cisplatin, GL-CDDP-Cy5.5 and
\end{abstract}

Correspondence to: Professor Yoshito Terai, Department of Obstetrics and Gynecology, Kobe University Graduate School of Medicine, 7-5-1 Kusunoki-cho Chuo-ku, Kobe, Hyogo 650-0017, Japan

E-mail: yterai@med.kobe-u.ac.jp

Abbreviations: EMT, epithelial-mesenchymal-transition; CDDP, cisplatin; GPI, glycosylphosphatidylinositol; DDS, drug delivery system; RES, reticuloendothelial system; HAS, human serum albumin; STR, short tandem repeat; Pt, platinum; MAC, maximum abdominal circumference

Key words: CD24, DDS, EMT, intraperitoneal xenograft models, ovarian cancer, drug delivery, platinum concentration, platinum resistance
CD24-GL-CDDP-Cy5.5 groups were 37, 36, 46 and 54 days after inoculation, respectively. Immunohistochemical analysis showed that CD24-GL-CDDP-Cy5.5 treatment, compared with GL-CDDP-Cy5.5, decreased the number of CD24-positive cells and suppressed the EMT phenomenon significantly $(\mathrm{P}<0.05)$. The present study demonstrated that CD24-GL-CDDP-Cy5.5, compared with other treatments, improved therapeutic efficacy. The present results suggested the potential for targeting anticancer therapeutics for CD24-positive cells to prevent disease progression.

\section{Introduction}

Ovarian carcinoma is the most frequent cause of cancer-related death from gynecologic malignancy and is frequently associated with a poor outcome due to the difficulty of an early diagnosis and malignancies which are often both large and advanced at the time of diagnosis (1). Almost $75 \%$ of women with ovarian cancer are diagnosed at stage III or IV, with 10 -year survival rates of $21 \%$ and less than $5 \%$, respectively (2). The degree of peritoneal dissemination and the number of chemotherapy-resistant tumors, as well, is related to the poor prognosis of patients with advanced-stage ovarian cancer. Epithelial-mesenchymal-transition (EMT) is associated with an important step in carcinoma metastasis via the induction of a highly invasive phenotype (3). We previously showed that the expression of EMT-related proteins was correlated with the status of tumor metastasis and the prognostic value in ovarian carcinoma (4). However, the molecular etiology of ovarian carcinoma remains mostly unknown. Therefore, it is of great importance to clarify the association of key proteins with ovarian carcinoma metastasis and invasion (5).

Cisplatin (CDDP) is widely used as an antineoplastic drug in the clinical treatment of ovarian cancer (6). However, its low aqueous solubility and high protein binding ability reduce the efficacy of the drug. In addition, its clinical use may be limited due to intrinsic and acquired resistance and systemic or nonspecific side effects, including acute nephrotoxicity and myelosuppresion $(7,8)$. Furthermore, the understanding of the pathways of cancer progression and pathogenesis, as well as the development of multidrug resistance in ovarian cancer, 
will result in the discovery of new molecular targets such as microRNA (9), somatic and germline mutations (10), amplifications (11), and structural instability (12). Moreover, new therapies such as drug delivery systems (DDS) also indicate more effective therapies into solid tumors while lessening their distribution into normal, healthy tissue (8). DDSs that can recognize specific tumors themselves, in order to deliver drugs at high doses efficiently and practically in vivo, have also been developed, and liposomes are being widely studied as DSS because of their amphiphilic characteristics (13).

Cluster of differentiation 24 (CD24) is a small, mucin-like glycosylphosphatidylinositol (GPI) anchored membrane molecule over-expressed in a variety of human carcinomas. CD24 is a glycoprotein expressed on the surface of most B lymphocytes and differentiating neuroblasts (14). In neoplasia, the expression of CD24 has not only been described in haematological malignancy, but also in a large variety of solid tumors such as nasopharyngeal carcinoma, non-small cell lung cancer, breast cancer, hepatocellular carcinoma, renal cell carcinoma, bladder carcinoma, colorectal cancer and epithelial ovarian cancer (15-17). Moreover, CD24 is involved in the growth, anchorage-independent reproduction and survival of tumor cells (18), and this finding suggests that CD24 functionally enhances the metastatic potential of cancer cells. In our previous study, we indicated that Caov-3 cells contain CD24-positive cells under normoxia and are involved in the EMT process, thus showing that CD24 plays a critical role in regulating the EMT phenomenon (19).

In the present study, we examined whether CD24-targeted liposomal CDDP specifically killed these CD24-positive cells, as CD24-positive cells are involved in both the EMT process and the suppression of tumor growth in Caov-3 xenograft mouse models. This study provides new knowledge on CD24-targeted therapeutic applications and their promise for future clinical trials.

\section{Materials and methods}

Properties of CD24 targeted liposomal cisplatin. Preparation of hydrophilic anionic liposomes and encapsulation of Cy5.5 were carried out as described previously $(20,21)$. These liposomes (GLYCOLIPO, Katayama Chemical Industries) are composed of dicetylphosphate, dipalmitoylphosphatidylcholine, dipalmitoylphosphatidylethanolamine, cholesterol and ganglioside. Dicetylphosphate was used to confer a negative charge to the liposome surface. Briefly, dipalmitoylphosphatidylcholine, cholesterol, ganglioside dicetylphosphate and dipalmitoylphosphatidylethanolamine were mixed at different molar ratios, and cholic acid was added to facilitate micelle formation. The mixture was dissolved in methanol/chloroform $(1: 1, v / v)$, and the solvent evaporated at $37^{\circ} \mathrm{C}$ to produce a lipid film which was dried under vacuum. This film was then dissolved in a $10 \mathrm{mM}$ TAPS buffer ( $N$-tris(hydroxymethyl) methyl-3-amino-propane sulfonic acid containing buffer) without $\mathrm{NaCl}$ at $\mathrm{pH} 8.4$ and sonicated to obtain a suspension of uniform micelles. Cy5.5 solution was then added to the micelle suspension, which was then ultrafiltered (molecular cutoff 10000$)$ (Amicon PM10). Tris(hydroxymethyl) aminomethane (Tris) was crosslinked on the liposome surface via bis(sulfosuccinimidyl)suberate (BS3) to confer hydrophilicity, because this process can prevent uptake by the reticuloendothelial system (RES) in the liver and spleen and by macrophages and vascular endothelial cells. Human serum albumin (HSA) and Cy5.5-NHS ester (GE Healthcare) were dissolved in TAPS ( $\mathrm{pH} \mathrm{8.4)} \mathrm{and} \mathrm{stirred} \mathrm{at} 37^{\circ} \mathrm{C}$ for $3 \mathrm{~h}$, and the HSA was then binded to Cy5.5. Using 3,30-dithiobis(sulfosuc cinimidylpropionate) (DTSSP, Pierce), anti-CD24 monoclonal antibody (Gene Copoeia) was crosslinked to HSA with Cy5.5, which was coupled in advance to the ganglioside component of the liposomes. DTSSP was used as a cross-linking reagent. Anti-CD24 was added to a final concentration of $75 \mu \mathrm{g} / \mathrm{ml}$ and stirred at $25^{\circ} \mathrm{C}$ for $2 \mathrm{~h}$. Tris- $\mathrm{HCl}$ was then added to reach a final concentration of $132 \mathrm{mg} / \mathrm{ml}$ and stirred overnight at $4^{\circ} \mathrm{C}$ for hydrophilization of the liposome surface. The content of lipid in the liposomes was determined as total cholesterol in the presence of $0.5 \%$ TritonX-100 with a Determiner TC555 diagnostic kit (Kyowa Medex). The particle size and zeta-potential were measured using a Zetasizer Nano-S90 (Malvern Instruments). The amount of anti-CD24 on the surface of the liposomes was measured by an enzyme-linked immunosorbent assay using a CD24-immobilized microplate as described previously (22). The final contents of both GL-CDDP-Cy5.5 and CD24-GL-CDDP-Cy5.5 had liposome concentrations of $72.0 \mathrm{mg} / \mathrm{ml}$ and CDDP concentrations of $2.0 \mathrm{mg} / \mathrm{ml}$, and both had a median diameter of $158 \mathrm{~nm}$.

Cell culture and cell lines. We used a human ovarian mucinous adenocarcinoma cancer cell line, Caov-3 cells, which was obtained from the American Type Culture Collection (ATCC) in this study. The ATCC routinely authenticate their cell lines by Short Tandem Repeat (STR) polymorphism profiling analyses. We also performed an STR polymorphism profiling analysis (Wakennyaku Co.) to confirm the cell line's identity. Caov-3 cells were grown in phenol DMEM containing $10 \%$ dextran-coated, charcoal-treated fetal calf serum in a humidified atmosphere of $5 \% \mathrm{CO}_{2}$ with $95 \%$ air at $37^{\circ} \mathrm{C}$.

Flow cytometry. The cultures from the Caov-3 cells were washed with PBS. For some experiments, single cells dissociated from tumor spheres were analyzed by the following method. One million trysinized cells were incubated with 7-amino-actinomycin D (BD Biosciences) only, or with 7-amino-actinomycin D and a mouse anti-human monoclonal antibody of CD24-FITC (BD Biosciences) with a stain buffer (BD Pharmingen $^{\mathrm{TM}}$ ) for $20 \mathrm{~min}$ at room temperature in the dark. After washing, the cells were analyzed using a BD FACS Aria $^{\mathrm{TM}}$. The fluorescence intensity was then analyzed using the BD FACS Diva software program (BD Biosciences).

Chemotherapeutic uptake and sensitivity assay in vitro. CDDP was encapsulated in GLYCOLIPO (GL-CDDP-Cy5.5), which was then conjugated with the anti-CD24 antibody (CD24-GL-CDDP-Cy5.5). The Caov-3 cells $\left(1 \times 10^{5}\right.$ cells/well) were seeded in triplicate in a 6 -well plate with GL-CDDP-Cy5.5 or CD24-GL-CDDP-Cy5.5. After $24 \mathrm{~h}$, the cells were washed with PBS, and we compared the differences in the uptake of Cy5.5 between GL-CDDP-Cy5.5 and CD24-GL-CDDP-Cy5.5 by fluorescence microscopy with a Biozero BZ-8100 (Keyence, Osaka, Japan). Flow cytometry was performed using the Caov-3 cells as previously described. 
The cells were then analyzed for FITC and Cy5 by a BD FACS Diva.

Intraperitoneal xenograft models of ovarian cancer. Female athymic nude mice (BALB/c Slc-nu/nu) were purchased from Japan SLC and maintained in accordance with the institutional guidelines of Osaka Medical College. All of the animal studies were carried out according to approved experimental protocols. $1.0 \times 10^{6}$ Caov-3 cells were suspended in $100 \mu \mathrm{l}$ PBS and were intraperitoneally injected into the nude mice (5-week-old). The ascites by peritoneal dissemination was allowed to grow to an approximately increased abdominal circumference of $30 \%$.

Platinum concentrations in tissue by ICP-OES. Thirty-six intraperitoneal xenograft models weighing 15-25 g were randomly divided into 4 groups ( $\mathrm{n}=9 /$ group). CDDP, GL-CDDP-Cy5.5 and CD24-GL-CDDP-Cy5.5 were injected intravenously with a single dose of $10 \mathrm{mg} / \mathrm{kg}$ body weight, and PBS served as the control group. The mice for each group were then sacrificed by an overdose of isoflurane 6,24 or $48 \mathrm{~h}(\mathrm{n}=3)$ after injection, and the disseminated tumors, livers and kidneys were dissected and washed with saline. The samples were then weighed (50 mg disseminated tumors, $100 \mathrm{mg}$ liver and $100 \mathrm{mg}$ kidney) and placed into the digestion vessels. The samples were digested with $1 \mathrm{ml} 65 \%$ nitric acid and $0.2 \mathrm{ml}$ hydrogen peroxide. After digestion and evaporation, the samples were poured into $10 \mathrm{ml}$ volumetric flasks which were filled up to the mark with bidistilled water. Two samples of disseminated tumors, liver and kidney were prepared from each animal. Absolute Platinum (Pt) concentrations in the tissue samples were measured by ICP-OES (Vista-MPX ICP-OES spectrometer, Seiko Instruments) as described previously (23). For the standardization of equipment and measurements, Spectro multi-element and Spectrum 3D standards were used (24). Standards were prepared in the same matrix as the samples. The samples were then measured 3 times, and blank subtraction was applied.

Computed tomography of intraperitoneal xenograft models to be treated. We employed a 3rd generation computed tomography scanner - LaTheta LCT-200 (Hitachi-Aloka). The tube voltage was set at $50 \mathrm{kV}$, and the current was constant at $0.5 \mathrm{~mA}$. The mice were scanned in a $48 \mathrm{~mm}$ wide specimen holder with a resolution of $96 \mu \mathrm{m}$ pixels. Whole abdominal scans in mice under the described conditions were 150 slices per mouse and a slice thickness of $192 \mu \mathrm{m}$. Mean acquisition time for the scan was $10 \mathrm{~min}$ and, therefore, the abdominal regions of the mice were scanned by LCT-200, as described previously, under isoflurane anesthesia (25).

Anti-tumor efficacy in vivo. Forty intraperitoneal xenograft models weighing 15-25 g were randomly assigned into four groups ( $\mathrm{n}=10$ per group) and intravascularly administered once a week with PBS, CDDP (10 mg/kg), GL-CDDP-Cy5.5 $(10 \mathrm{mg} / \mathrm{kg})$, or CD24-GL-CDDP-Cy5.5 (10 mg/kg) for 3 weeks. Maximum abdominal circumference (MAC), due to the increased ascites, was measured by a $3 \mathrm{D}$ imaging computed tomography scanner (LCT-200) once a week as a base to evaluate the therapeutic efficacy. The mice were also measured by LCT-200 once a week after treatment, and the increase rate of MAC was calculated. Tumor growth curves were stopped at the exclusion day of the first mouse in each group. The survival benefit by treatment was measured with the calculation of overall survival days, and Kaplan-Meier survival plots were recorded.

In order to determine the survival of the disseminated tumor-bearing nude mice, the mice were checked daily for survival and were sacrificed, according to our institutional guidelines, when the abdominal circumference had reached $10 \mathrm{~cm}$.

Immunohistochemical analysis of intraperitoneal xenograft models. The disseminated samples with intravenously injected PBS, CDDP, GL-CDDP-Cy5.5 or CD24-GL-CDDP-Cy5.5 were formalin-fixed and embedded in paraffin. Deparaffinized and rehydrated sections $(4-\mu \mathrm{m})$ were autoclaved in $0.01 \mathrm{~mol} / \mathrm{l}$ citrate buffer ( $\mathrm{pH} \mathrm{6.0)}$ for $15 \mathrm{~min}$ at $121^{\circ} \mathrm{C}$ for antigen retrieval. Endogenous peroxidase activity was blocked with $0.3 \%$ solution hydrogen peroxidase in methanol for $30 \mathrm{~min}$. Tumor sections were incubated at $4^{\circ} \mathrm{C}$ for $12 \mathrm{~h}$ with a CD24-specific antibody (1:50 dilution; Thermo Fisher Scientific, Inc.), and E-cadherin (1:50 dilution; Cell Signaling Technology) and Snail antibody (1:100 dilution; ABGENT) were used as described in a previous study $(4,19)$. The sections were washed with $1 \mathrm{X}$ phosphate-buffered saline (PBS) and incubated with Histofine Simple Stain MAX PO (Multi; Nichirei) for $30 \mathrm{~min}$ at room temperature. Finally, the sections were washed with 1X PBS, and the signals were visualized by incubation with $\mathrm{H}_{2} \mathrm{O}_{2}$ /diaminobenzidine substrate solution for $5 \mathrm{~min}$. The sections were then counterstained with hematoxylin prior to dehydration and mounting.

Immunohistochemical evaluations. The expression levels of CD24, E-cadherin and Snail were assessed using a semiquantitative system, ranging from $0,1+, 2+$ to $3+$. Briefly, the expression of CD24 was assessed as carcinoma cells with positive staining in the membrane and/or cytoplasm and scored as follows: 0 , negative; $1+,<10 \%$ positive cells; $2+, 10-50 \%$ positive cells; $3+$, $>50 \%$ positive cells. The E-cadherin expression was scored as follows: 0 , negative or $<10 \%$ immunoreactivity of tumor cells; $1+,>10 \%$ low-intensity immunoreactivity of tumor cells, $2+$, $>10 \%$ medium-intensity immunoreactivity of tumor cells, $3+$, $>10 \%$ high-intensity immunoreactivity of the tumor cells. The Snail expression was evaluated as being positive only when nuclear staining was detectable, as follows: 0 , negative or $<1 \%$ positive cells; $1+,>1 \%$ positive cells; $2+, 2-5 \%$ positive cells; $3+,>5 \%$ positive cells. Samples with heterogeneous staining were scored based on the intensity in the largest stained area. Tumor-infiltrating immune cells were evaluated as described in a previous study $(26,27)$.

Statistical analysis. All statistical analyses were performed using the JMP software program (SAS Institute). Continuous variables are expressed as the median and interquartile range or the mean \pm standard deviation. Dunnett's test was used to compare continuous variables. Statistical differences were evaluated using the Kruskal-Wallis test prior to Dunnett's post hoc test. The univariate analyses of overall survival were determined according to the Kaplan-Meier method using the log-rank test, and the statistical analysis of post hoc test were 
used by Dunnett's test. Descriptive statistics and 95\% confidence intervals (CI) were built to proportions of all groups. $\mathrm{P}<0.05$ was considered to indicate a statistically significant difference.

\section{Results}

Therapeutic effect of CD24-targeted liposomal cisplatin in vitro. At FITC values, ovarian cancer cell line Caov-3 cells were divided into about $30 \%$ CD24-positive cells and about $70 \%$ CD24-negative cells (Fig. 1). We confirmed that it is quite reasonable to select CD24 to target ovarian cancer as an application of a specific DDS. To clarify whether the CD24-targeted liposomal cisplatin (CD24-GL-CDDP-Cy5.5) has more sensitivity on Caov-3 cells than the non-targeted liposome containing cisplatin (GL-CDDP-Cy5.5; Fig. 2A), we used BZ-8100 fluorescence microscopy after the treatment. The uptake of Cy5.5 in Caov-3 cells was not different between treatment with GL-CDDP-Cy5.5 and with CD24-GL-CDDP-Cy5.5 (Fig. 2B). Subsequently, we performed flow cytometry on the Caov-3 cells with each treatment. Fig. 2C shows that CD24-GL-CDDP-Cy5.5 accumulates more Cy5 in Caov-3 cells with higher FITC values; CD24-GL-CDDP-Cy5.5 was uptaken more in CD24-positive cells than in GL-CDDP-Cy5.5 in vitro. However, we examined the sensitivity of the cell viability of among CDDP, GL-CDDP-Cy5.5 and CD24-GL-CDDP-Cy5.5 administrations in Caov-3 ovarian cancer cells, including CD24+ and CD24-, using an MTS assay. CDDP, GL-CDDP-Cy5.5 and CD24-GL-CDDP-Cy5.5 acted as a concentration dependent inhibitor of cell proliferation with an IC50 of 72.4, 78.7 and $76.7 \mu \mathrm{M}$, respectively. There was no significant difference in chemotherapeutic sensitivity among CDDP, GL-CDDP-Cy5.5 and CD24-GL-CDDP-Cy5.5 in Caov-3 ovarian cancer cells (Fig. S1).

Platinum concentrations in various tissue samples of nude mice by ICP-OES. Progression in tumorigenesis of ovarian cancer is usually intraperitoneal dissemination. We examined whether CD24-GL-CDDP-Cy5.5 is specifically uptaken to intraperitoneal xenograft models by measuring the Pt concentration contained in cisplatin. $1.0 \times 10^{6} \mathrm{CaOv}-3$ cells were inoculated intraperitoneally in nude mice, as described in Materials and Methods. The intraperitoneal xenograft models with peritoneal fluid were classified into several treatment groups, including PBS, CDDP, GL-CDDP-Cy5.5 and CD24-GL-CDDP-Cy5.5. Furthermore, each group was distinguished by the time at which each agent was injected (6h, 24h or $48 \mathrm{~h}$ ). Absolute Pt concentrations in disseminated tumors, kidney and liver of the nude mice after sacrificing were measured by ICP-OES. The concentration of $\mathrm{Pt}$ was below the limit of detection in the group treated with PBS, while Pt concentration was detectable in the group treated with CDDP, GL-CDDP-Cy5.5 and CD24-GL-CDDP-Cy5.5. The $\mathrm{Pt}$ concentration in dissemination tumors increased in treated groups after $6 \mathrm{~h}$. The Pt concentration in dissemination tumors treated with CD24-GL-CDDP-Cy5.5 after $48 \mathrm{~h}$ was significantly higher than that treated with CDDP $(36.5 \pm 6.5 \mu \mathrm{g} / \mathrm{mg}$ vs. $13.2 \pm 4.3 \mu \mathrm{g} / \mathrm{mg}, \mathrm{P}<0.05$; Fig. 3A), however, there was no difference in the $\mathrm{Pt}$ concentration between the GL-CDDP-Cy5.5 and CD24-GL-CDDP-Cy5.5 groups. Fig. 3B shows the transition of $\mathrm{Pt}$ concentration over time in the kidney.

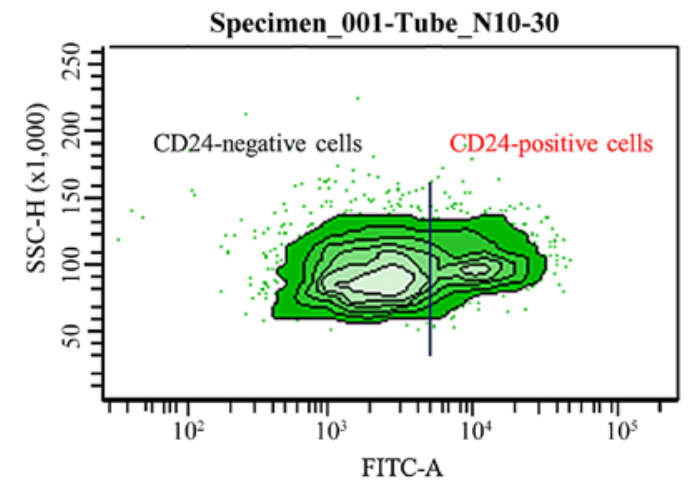

Figure 1. Caov-3 cells contain CD24-positive cells and CD24-negative cells. Caov-3 is classified into CD24-positive cells and negative cells with the fluorescence intensity of FITC by flow cytometry. FITC, fluorescein isothiocyanate.

After 6 and $24 \mathrm{~h}$, the Pt concentration immediately lower in both the GL-CDDP-Cy5.5 and CD24-GL-CDDP-Cy5.5 groups than in the CDDP group, and the Pt concentration in kidneys treated with CDDP after $48 \mathrm{~h}$ was significantly higher than in those treated with CD24-GL-CDDP-Cy5.5 $(114.4 \pm 36.1 \mu \mathrm{g} / \mathrm{mg}$ vs. $46.5 \pm 10.1 \mu \mathrm{g} / \mathrm{mg}, \mathrm{P}<0.05)$; however, there was no difference in the Pt concentration between the GL-CDDP-Cy5.5 and CD24-GL-CDDP-Cy5.5 groups. These finding suggest that the GL-CDDP-Cy5.5 and CD24-GL-CDDP-Cy5.5 markedly reduced nephrotoxicity, which is a dose-limiting factor of CDDP. Fig. 3C shows the transition of $\mathrm{Pt}$ concentration over time in the liver. In contrast, the Pt concentration in livers treated with CD24-GL-CDDP-Cy5.5 after $48 \mathrm{~h}$ was significantly higher than in those treated with CDDP $(78.5 \pm 21.7 \mu \mathrm{g} / \mathrm{mg}$ vs. $38.5 \pm 8.3 \mu \mathrm{g} / \mathrm{mg}$, $\mathrm{P}<0.05)$; however, there was no difference in the Pt concentration between the GL-CDDP-Cy5.5 and CD24-GL-CDDP-Cy5.5 groups. Although the Pt concentration in liver tissues from the GL-CDDP-Cy5.5 and CD24-GL-CDDP-Cy5.5 groups was higher compared with that in the CDDP group, the liver pathological changes of microvesicular steatosis and inflammatory infiltrates were hardly observed (data not shown).

Antitumor efficiency of CD24-GL-CDDP-Cy5.5 in intraperitoneal xenograft models. A total of 40 nude mice were intraperitoneally inoculated with Caov-3 cells and followed up for 7 days for tumor growth. The nude mice were treated with PBS, CDDP (10 mg/kg), GL-CDDP-Cy5.5 (10 mg/kg) or CD24-GL-CDDP-Cy5.5 (10 mg/kg) once a week. Each agent was injected via the tail vein on day 8,15 and 22 . On day 29, the MAC was measured by LCT-200 and was significantly different between the PBS group and the CD24-GL-CDDP-Cy5.5 group (Fig. 4A). The mice which were injected with PBS and CDDP showed a fast increase in ascites volume from the beginning of the therapy experiment, thus indicating an uninhibited MAC growth (Fig. 4B). On day 22, the MAC growth value was 1.62 in PBS, 1.65 in CDDP, 1.45 in GL-CDDP-Cy5.5, and 1.26 in CD24-GL-CDDP-Cy5.5. The group that received CD24-GL-CDDP-Cy5.5 demonstrated the most pronounced delay in MAC growth (vs. PBS: $\mathrm{P}<0.01$; vs. CDDP: $\mathrm{P}<0.05$; vs. GL-CDDP-Cy5.5: $\mathrm{P}<0.05)$. The antitumor effects of CD24-GL-CDDP-Cy5.5, as well, were also evaluated by the survival of mice in the same experiments 
A

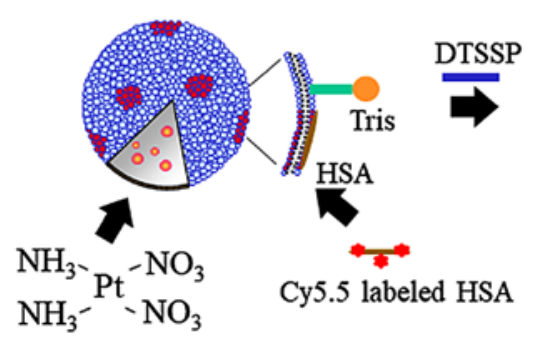

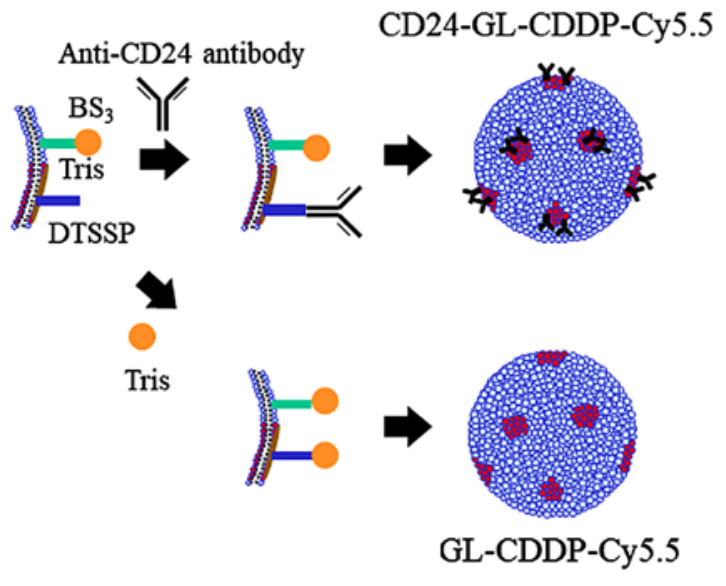

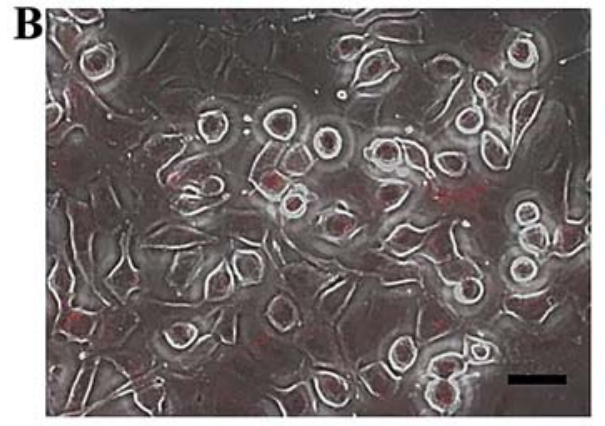

GL-CDDP-C y 5.5

C

GL-CDDP-C y 5.5

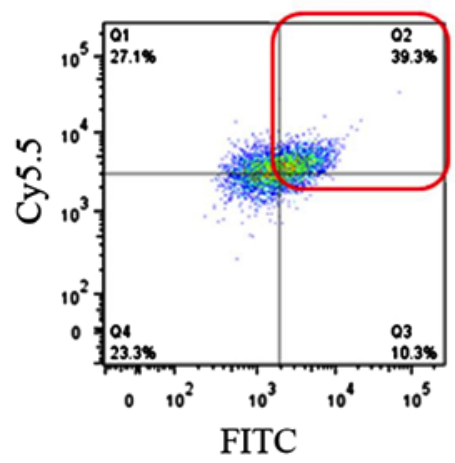

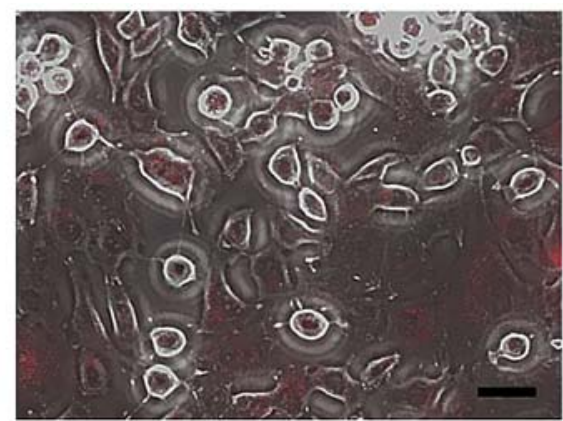

CD24-GL-CDDP-C y 5.5

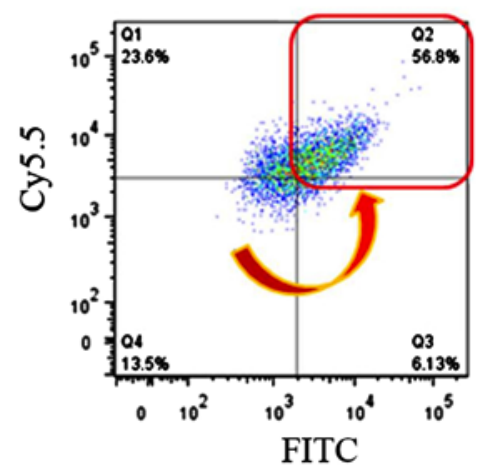

Figure 2. Preparation scheme of the liposome with antibody. (A) CDDP was encapsulated in the liposome. DTSSP was used as a cross-linking reagent to anti-CD24 antibody (CD24-GL-CDDP-Cy5.5). Tris-HCl was then added for hydrophilization of the liposome surface. The liposome without modified anti-CD24 antibody was GL-CDDP-Cy5.5. (B) In total, 1.0x10 ${ }^{6}$ Caov-3 cells were cultured with $100 \mu 1$ GL-CDDP-Cy5.5 or CD24-GL-CDDP-Cy5.5 for $24 \mathrm{~h}$ The red fluorescence specifies the localization of the liposomes on Caov-3. (C) CD24-GL-CDDP-Cy5.5 is highly accumulated with Cy5 in CD24 positive-cells as compared with GL-CDDP-Cy5.5. Scale bar, $100 \mu \mathrm{m}$. HSA, human serum albumin; BS3, bis(sulfosuccinimidyl) suberate; DTSSP, 3,3-dithiobis(sulfosuccin imidylpropionate); FITC, fluorescein isothiocyanate; CDDP, cisplatin; Cy5.5, cyanine 5.5.

in vivo (Fig. 4C). The ten mice of each groups treated with PBS, CDDP, GL-CDDP-Cy5.5 or CD24-GL-CDDP-Cy5.5 died between day 24 and 96 . The median survival time was 36 days in the PBS group (95\% CI=23-48), 37 days in the CDDP group (95\% CI=23-49), 46 days in the GL-CDDP-Cy5.5 group (95\% CI=29-50) and 56 days in the CD24-GL-CDDP-Cy5.5 group (95\% CI=32-60). No significant difference in overall survival could be observed between the PBS, CDDP and GL-CDDP-Cy5.5 groups. Moreover, the CD-24-GL-CDDP-Cy5.5 group had a significantly elongated survival period of mice, as compared with the PBS $(\mathrm{P}<0.05)$ and CDDP $(\mathrm{P}<0.05)$ group, but not the GL-CDDP-Cy5.5 group $(P=0.2)$. Additionally, no weight loss $>20 \%$ or signs of distress could be observed at any time during the therapy experiment. These results supported the superior antitumor efficacy observed with CD24-GL-CDDP-Cy5.5 in vivo.

CD24-GL-CDDP-Cy5.5 suppress EMT phenomenon. Immunohistochemical staining showed that the number of CD24-positive cells was significantly lower in the CD24-GL-CDDP-Cy5.5 group compared to the untreated control group $(\mathrm{P}<0.05)$, CDDP group $(\mathrm{P}<0.05)$, or the GL-CDDP-Cy5.5 group $(\mathrm{P}<0.05)$ (Fig. 5A and $\mathrm{B})$. The expression of E-cadherin was significantly higher in the CD24-GL-CDDP-Cy5.5 group than in the other groups $(\mathrm{P}<0.05)$. The expression of Snail was also significantly lower 

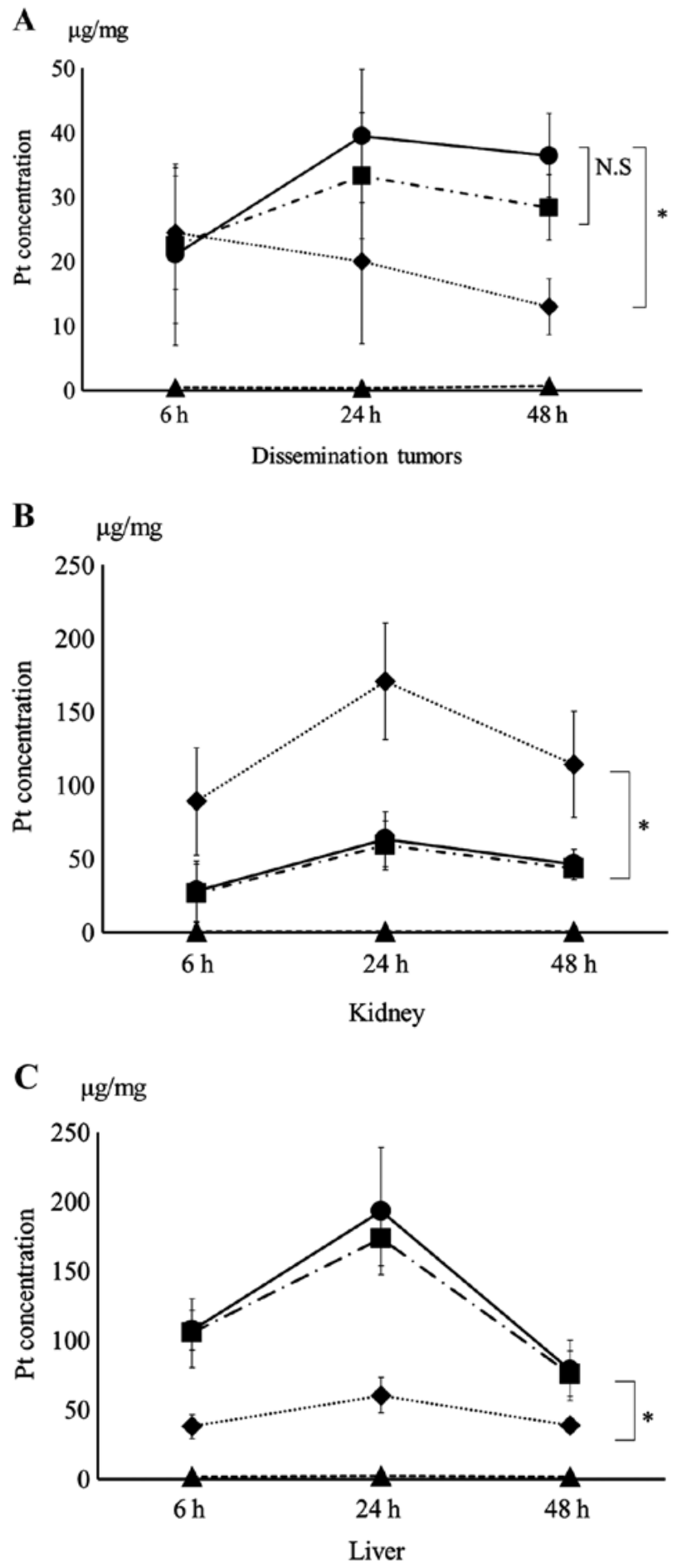

Figure 3. Pt concentrations in disseminated tumors, kidney and liver of intraperitoneal xenograft models. Pt concentrations $(\mu \mathrm{g} / \mathrm{mg} \pm \mathrm{SD})$ in the tissue samples of PBS, CDDP, GL-CDDP-Cy5.5 or CD24-GL-CDDP-Cy5.5 treated nude mice (single dose of $10 \mathrm{mg} / \mathrm{kg}$ body weight) at 6,24 or $48 \mathrm{~h}$ after administration. After $48 \mathrm{~h}$, Pt concentrations in (A) disseminated tumors, (B) the kidney or (C) the liver were determined. The triangles represent the PBS group; the diamonds represent the CDDP group; the squares represent the GL-CDDP-CY5.5 group; and the circles represent the CD24-GL-CDDP-CY5.5 group. P-values were calculated using the Dunnett's test. ${ }^{*}<0.05$. NS, not significant; Pt, platinum; CDDP, cisplatin.

in the CD24-GL-CDDP-Cy5.5 group than that in the other groups $(\mathrm{P}<0.05$; Fig. 5B). These results supported CD24 targeted liposomes' ability to suppress the EMT phenomenon in vivo.

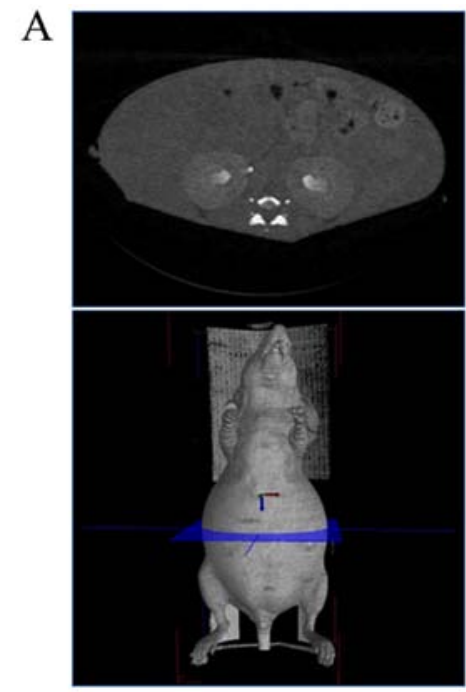

PBS

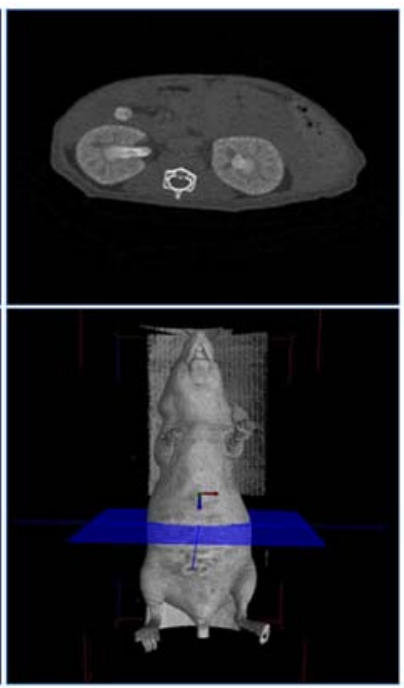

CD24-GL-CDDP-CY5.5
B
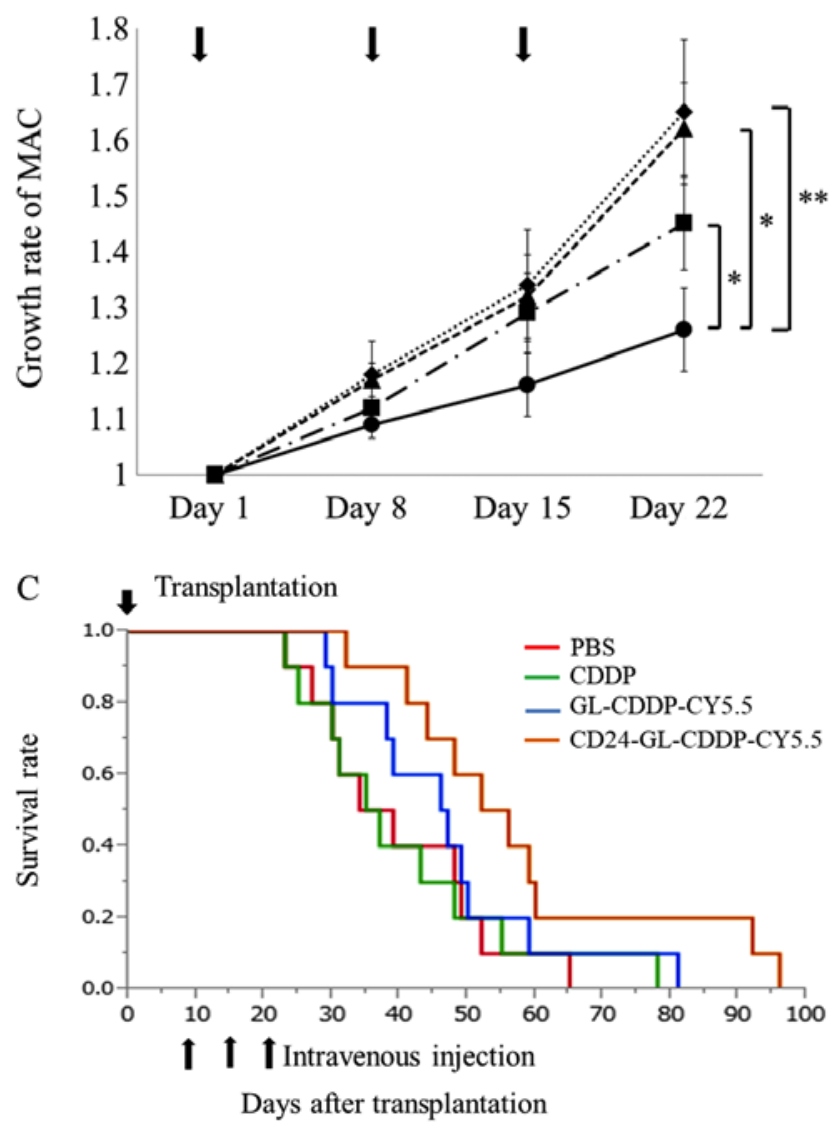

Figure 4. Therapeutic efficacy of anti-CD24 targeted liposomal cisplatin. Intraperitoneal xenograft models received PBS $(n=10), \operatorname{CDDP}(\mathrm{n}=10)$, GL-CDDP-Cy5.5 ( $\mathrm{n}=10)$ and CD24-GL-CDDP-Cy5.5 ( $\mathrm{n}=10)$ once a week for a total of three times. (A) Representative 3D imaging of mice treated with PBS and CD24-GL-CDDP-Cy5.5 on day 22. (B) Calculated mean relative growth rate of MAC in mice scanned by LCT-200. The triangles represent the PBS group; the diamonds represent the CDDP group; the squares represent the GL-CDDP-CY5.5 group; and the circles represent the CD24-GL-CDDP-CY5.5 group. P-values were calculated using the Dunnett's test. ${ }^{*} \mathrm{P}<0.05,{ }^{* *} \mathrm{P}<0.01$. (C) Survival curves generated using the Kaplan-Meier method in 40 intraperitoneal xenograft models. The CD-24-GL-CDDP-Cy5.5 group had a significantly longer survival period of mice compared with the PBS $(\mathrm{P}<0.05)$ and $\mathrm{CDDP}(\mathrm{P}<0.05)$ groups, but not the GL-CDDP-Cy5.5 group $(\mathrm{P}=0.2)$. There was no significant difference observed in overall survival between the PBS, CDDP and GL-CDDP-Cy5.5 groups. P-values were calculated using the Dunnett's test. CDDP, cisplatin. 
$\mathbf{A}$

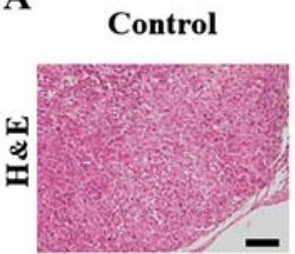

CDDP
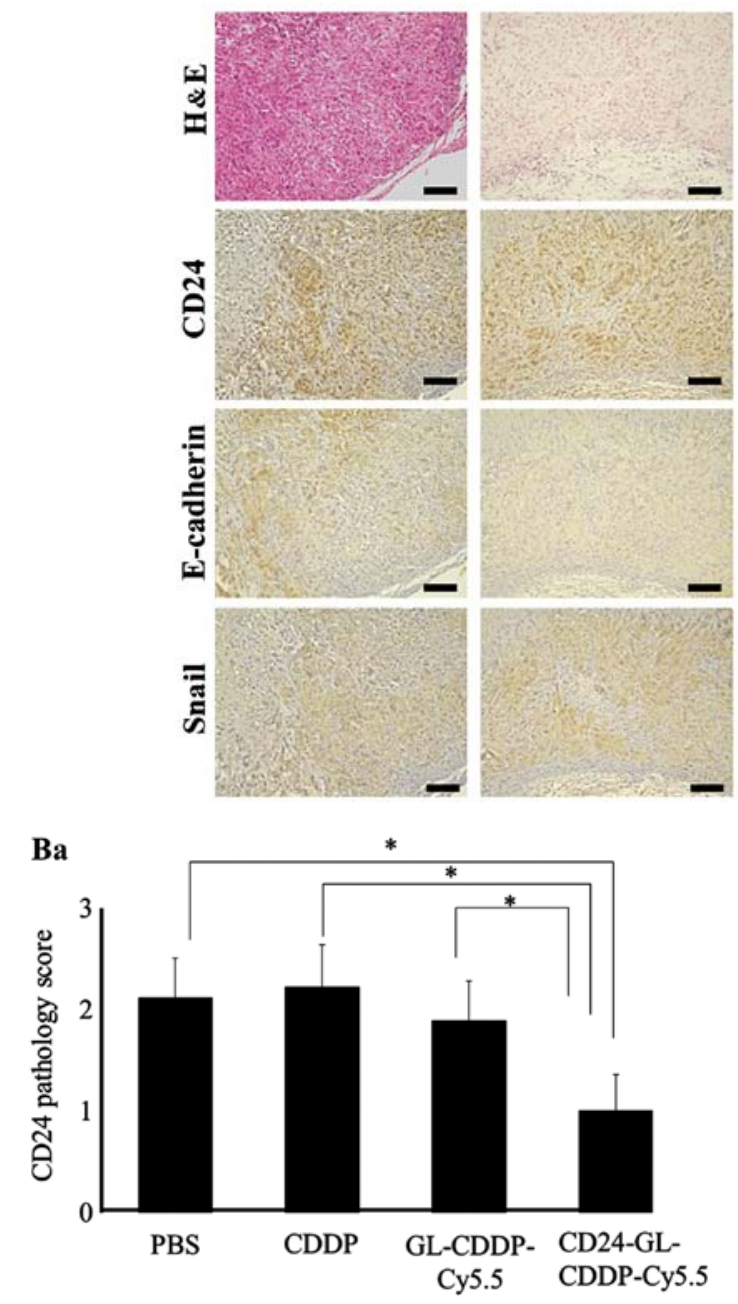

$-$

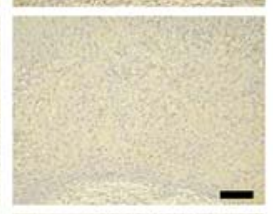

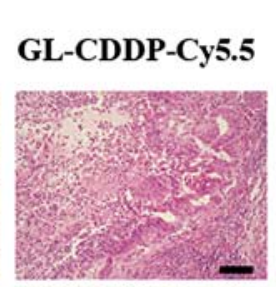

CD24-GL-CDDP-

Cy5.5
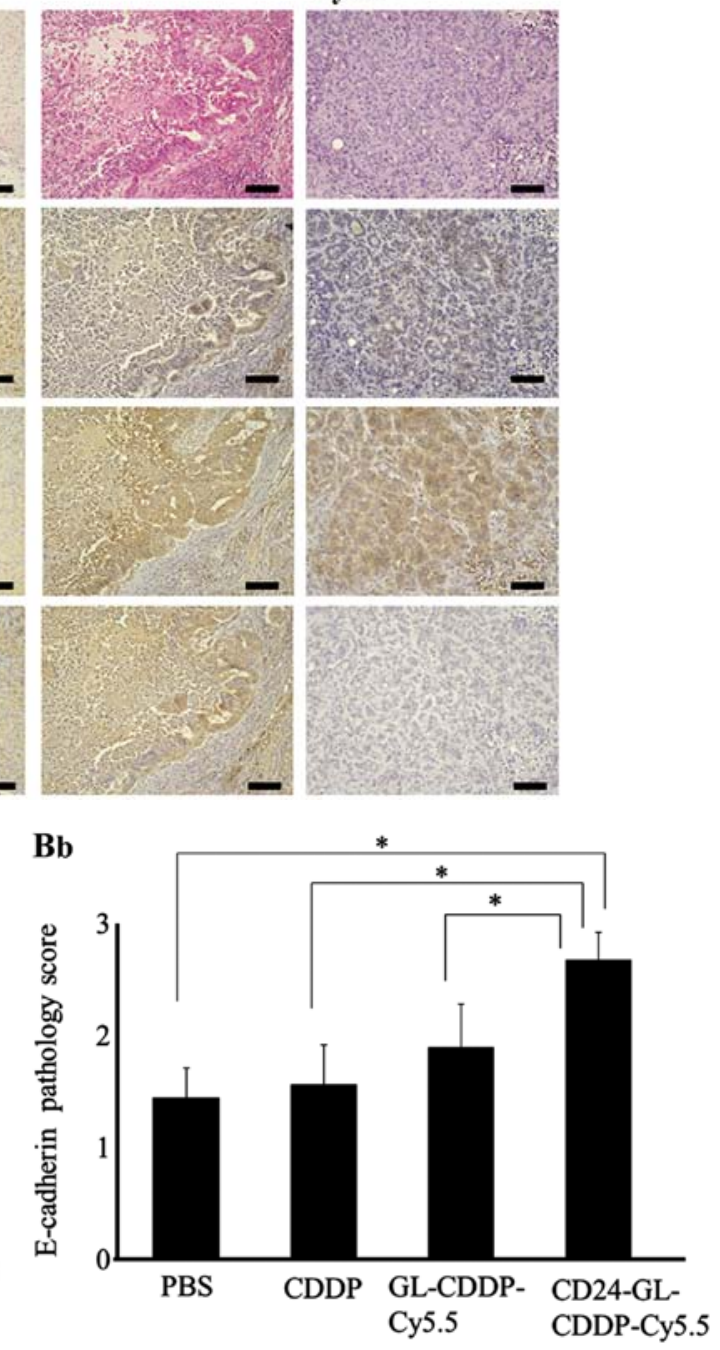

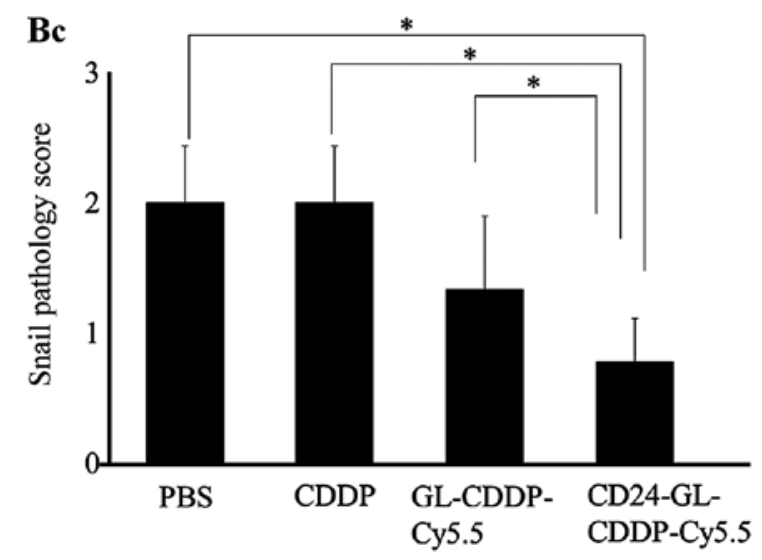

Figure 5. Immunohistochemical analysis of Caov-3 tumor bearing mice. (A) Representative immunohistological images of tumor tissues showing CD24, E-cadherin and Snail staining after different treatments. CD24-GL-CDDP-Cy5.5 reduced the expression of CD24 and Snail and enhanced the expression of E-cadherin in the disseminated tumors. (Ba) CD24-GL-CDDP-Cy5.5 significantly reduced CD24 positive cells compared with other treatments. (Bb) CD24-GL-CDDP-Cy5.5 significantly increased E-cadherin compared with other treatments. (Bc) CD24-GL-CDDP-Cy5.5 significantly reduced Snail compared with other treatments. Scale bar, $100 \mu \mathrm{m}$. " P<0.05. CDDP, cisplatin.

\section{Discussion}

In the present study, the possibility of DDS targeting CD24 for ovarian cancer was assessed. Antibody drug conjugates use the antibody as a guided missile to deliver cytotoxic molecules to tumor cells to achieve cancer therapy. Critical parameters for antibody drug conjugates include the selection of the tumor target antigen, the antibody against the target, the cytotoxic molecule, the linker bridging the cytotoxic molecule, and the antibody which aided the successful development of DDSs for 
clinical application. The target antigen is the initial point at which to design a DDS, and many tumor specific targets have been evaluated for development. CD24 is an important marker for the diagnosis and prognosis of various cancers (15-17). We previously reported that CD24 was expressed in $70.1 \%$ of ovarian cancers and that the expression of CD24 was an independent predictor of patient survival in patients with ovarian cancer (19). We also demonstrated that the overexpression of CD24 in ovarian carcinoma is associated with high invasiveness and metastatic potential, high tumor proliferation status, and the activation of both the Akt and ERK pathways. Moreover, CD24 is a novel predictor for poor prognosis and induces the EMT phenomenon in ovarian carcinoma $(19,28)$. EMT is involved in cell invasion, resistance to chemotherapy, and the formation of side populations of cancer stem-like cells (CSCs) (29), and we demonstrated that CD24-positive cells have characteristic of CSCs in a previous study (19). We have also indicated that $\mathrm{CD} 24$ plays a critical role in regulating the EMT phenomenon in ovarian cancer (19). Therefore, we believe that CD24 is the best target of an organotropic drug delivery system in advanced ovarian cancer and, thus, developed a CD24-targeted liposomal CDDP incorporating micells. In the present study, CDDP as the classical chemotherapeutic drug was selected to be coupled with an anti-CD24 antibody on GLYCOLIPO, and CD24-GL-CDDP-Cy5.5 showed a targeting potency against CD24-positive cells in vitro. Based on this, we investigated the effectiveness of CD24-GL-CDDP-Cy5.5 using a xenograft mouse model with resistance to CDDP. Studies of tumor inhibition are often done over a short time interval, which is insufficient to study tumor invasion in vivo. To avoid this limitation, we investigated the effect of drug treatments for over a month post treatment. The intravenous injection of CD24-GL-CDDP-Cy5.5 treatment effectively decreased tumor growth after treatment. In this study, nude mice received only three doses of CD24-GL-CDDP-Cy5.5, and it is possible that additional doses could have further limited tumor growth.

CD24-positive cells have CDDP resistance, but acertain effect can be obtained with a higher concentration of CDDP in vitro (19). In this study, we showed the ability of GL-CDDP-Cy5.5 and CD24-GL-CDDP-Cy5.5 to promote significantly higher $\mathrm{Pt}$ concentrations in disseminated tumors than free CDDP through intra-vein injection after $48 \mathrm{~h}$. It is well known that long-circulating carriers, such as GLYCOLIPO, are able to increase drug accumulation in tumors due to the enhanced permeability and retention effect (30). In the disseminated tumors, there was no significant difference between GL-CDDP-Cy5.5 and CD24-GL-CDDP-Cy5.5 in terms of Pt concentration. This result is presumed to be due to the coexistence of CD24-positive cells and CD24-negative cells in disseminated tumors. Flow cytometry showed that CD24-GL-CDDP-Cy5.5 increased the accumulation of Cy5 dye in CD24-positive cells specifically. It was suggested that CD24-GL-CDDP-Cy5.5 was uptaken with a higher concentration of CDDP in CD24-positive cells than was GL-CDDP-Cy5.5. Immunohistochemical evaluation of dissemination tumors demonstrated that CD24-GL-CDDP-Cy5.5 reduced the expression of CD24 more than PBS, CDDP and GL-CDDP-Cy5.5. It was also suggested that CD24-GL-CDDP-Cy5.5 had a higher CDDP concentration in CD24-positive cells and reduced the number of the cells compared with the other groups. In this study, we demonstrated that the Pt concentration in dissemination tumors treated with CD24-GL-CDDP-Cy5.5 maintained a higher Pt concentration than that in the CDDP group, and that it was significantly different after $48 \mathrm{~h}(36.5 \pm 6.5 \mu \mathrm{g} / \mathrm{mg}$ vs. $13.2 \pm 4.3 \mu \mathrm{g} / \mathrm{mg}, \mathrm{P}<0.05)$. In contrast, the Pt concentration in the kidney immediately lower in the GL-CDDP-Cy5.5 and CD24-GL-CDDP-Cy5.5 groups than in CDDP group $(43.7 \pm 8.3 \mu \mathrm{g} / \mathrm{mg}$ and $46.5 \pm 10.1 \mu \mathrm{g} / \mathrm{mg}$ vs. $114.4 \pm 36.1 \mu \mathrm{g} / \mathrm{mg}, \mathrm{P}<0.05)$. As the nephrotoxicity of CDDP is considered to depend on the peak urinary Pt concentration (31), we demonstrated that CD24-GL-CDDP-Cy5.5 has not only the potential for maintaining a higher $\mathrm{Pt}$ concentration in dissemination tumors but also in reducing nephrotoxicity. In the other words, CD24-GL-CDDP-Cy5.5 has the ability for a safer administration in ovarian cancer patients. However, further pre-clinical studies using other animal models are necessary.

Snail is a transcriptional repressor of E-cadherin during the EMT phenomenon (32). Snail expression in peritoneal dissemination is associated with an unfavorable prognosis in ovarian cancer (33). CD24-GL-CDDP-Cy5.5 reduced the expression of Snail and enhanced the expression of E-cadherin. In previous a study, we suggested that CD24 is a key molecule of metastatic progression in the EMT phenomenon (19). This study also showed that CD24-GL-CDDP-Cy5.5 can suppress the EMT phenomenon by decreasing CD24 expression. Due to this suppression of the EMT phenomenon, the decrease in MAC in intraperitoneal xenograft models indicates that the anti-cancer efficacy of CD24-GL-CDDP-Cy5.5 is better in comparison with PBS, CDDP and GL-CDDP-Cy5.5. In addition, CD24-GL-CDDP-Cy5.5 significantly prolonged the survival rate of Caov-3 bearing mice, in comparison with PBS, CDDP and GL-CDDP-Cy5.5. Thus, our data suggests that CD24-GL-CDDP-Cy5.5 contributes to the suppression of the EMT phenomenon in intraperitoneal xenograft models transplanted with the Caov-3 cell line.

In conclusion, the present results indicat that the CD24-GL-CDDP-Cy5.5 we generated is a selective targeted to CD24-positive ovarian carcinoma cells. Moreover, CD24-GL-CDDP-Cy5.5 can suppress the EMT phenomenon. Taken together, this study shows that CD24-GL-CDDP-Cy5.5 is effective for aggressive ovarian cancer that has acquired a resistant to CDDP, although there are required that the future studies should examine the effect of CD24 targeted liposomes in models with different levels of CD24 expression, and that side effects of treatment with CD24 targeted liposomes must be assessed in future studies. We consider that our strategy of targeting CD24 is promising for clinical applications.

\section{Acknowledgements}

Not applicable.

\section{Funding}

The present study was supported by a Japan Society for the Promotion of Science KAKENHI grant (grant no. 16K11162 to YTe).

\section{Availability of data and materials}

Not appicable. 


\section{Authors' contributions}

$\mathrm{KA}$, YTe and MO were involved in study conception and design, data analysis, drafting the article and its final edition. KA, TT, YTa, SF, KM, ST, HS and MH performed sample collection and performed all in vivo experiments. KA, TT, $\mathrm{KM}$ and $\mathrm{MH}$ analyzed data. KA, YTe and MO wrote the manuscript and checked all data. All authors gave their final approval of the submitted version.

\section{Ethics approval and consent to participate}

The present study was approved by the Institutional Review Board of Osaka Medical College.

\section{Patient consent for publication}

Not applicable.

\section{Competing interests}

The authors declare that they have no competing interests.

\section{References}

1. Siegel R, Naishadham D and Jemal A. Cancer statistics, 2013. CA Cancer J Clin 63: 11-30, 2013.

2. Jelovac D and Armstrong DK: Recent progress in the diagnosis and treatment of ovarian cancer. CA Cancer J Clin 61: 183-203, 2011.

3. Thiery JP: Epithelial-mesenchymal transitions in tumour progression. Nat Rev Cancer 2: 442-454, 2002.

4. Takai M, Terai Y, Kawaguchi H, Ashihara K, Fujiwara S, Tanaka T, Tsunetoh S, Tanaka Y, Sasaki H, Kanemura M, et al: The EMT (epithelial-mesenchymal-transition)-related protein expression indicates the metastatic status and prognosis in patients with ovarian cancer. J Ovarian Res 7: 76, 2014.

5. Liu Z, Yun R, Yu X, Hu H, Huang G, Tan B and Chen T: Overexpression of Notch 3 and $\mathrm{pS} 6$ is associated with poor prognosis in human ovarian epithelial cancer. Mediators Inflamm 2016: 5953498, 2016.

6. Loehrer PJ and Einhorn LH: Cisplatin. Ann Intern Med 100: 704-713, 1984.

7. Kartalou M and Essigmann JM: Mechanisms of resistance to cisplatin. Mutat Res 478: 23-43, 2001.

8. Uchino H, Matsumura Y, Negishi T, Koizumi F, Hayashi T, Honda T, Nishiyama N, Kataoka K, Naito S and Kakizoe T: Cisplatin-incorporating polymeric micelles (NC-6004) can reduce nephrotoxicity and neurotoxicity of cisplatin in rats. Br J Cancer 93: 678-687, 2005.

9. Lu L, Schwartz P, Scarampi L, Rutherford T, Canuto EM, Yu H and Katsaros D: MicroRNA let-7a: A potential marker for selection of paclitaxel in ovarian cancer management. Gynecol Oncol 122: 366-371, 2011.

10. Cancer Genome Atlas Research Network: Integrated genomic analyses of ovarian carcinoma. Nature 474: 609-615, 2011.

11. Patch AM, Christie EL, Etemadmoghadam D, Garsed DW George J, Fereday S, Nones K, Cowin P, Alsop K, Bailey PJ, et al: Whole-genome characterization of chemoresistant ovarian cancer. Nature 521: 489-494, 2015.

12. Lalwani N, Prasad SR, Vikram R, Shanbhogue AK, Huettner PC and Fasih N: Histologic, molecular, and cytogenetic features of ovarian cancers: Implications for diagnosis and treatment. Radiographics 31: 625-646, 2011.

13. Hwang SY, Cho DY, Kim HK, Cho SH, Choo J, Yoon WJ and Lee EK: Preparation of targeting proteoliposome by postinsertion of a linker molecule conjugated with recombinant human epidermal growth factor. Bioconjug Chem 21: 345-351, 2010.

14. Phillips TM, McBride WH and Pajonk F: The response of CD24(-/low)/CD44+ breast cancer-initiating cells to radiation. J Natl Cancer I 98: 1777-1785, 2006.
15. Kristiansen G, Denkert C, Schlüns K, Dahl E, Pilarsky C and Hauptmann S: CD24 is expressed in ovarian cancer and is a new independent prognostic marker of patient survival. Am J Pathol 161: 1215-1221, 2002.

16. Kristiansen G, Winzer KJ, Mayordomo E, Bellach J, Schlüns K, Denkert C, Dahl E, Pilarsky C, Altevogt P and Guski H. CD24 expression is a new prognostic marker in breast cancer. Clin Cancer Res 9: 4906-4913, 2003.

17. Zhu J, Zhang G and Lu H: CD24, COX-2, and p53 in epithelial ovarian cancer and its clinical significance. Front Biosci (Elite Ed) 4: 2645-2651, 2012.

18. Lee TK, Castilho A, Cheung VC, Tang KH, Ma S and Ng IO: CD24(+) liver tumor-initiating cells drive self-renewal and tumor initiation through STAT3-mediated NANOG regulation. Cell Stem Cell 9: 50-63, 2011.

19. Nakamura K, Terai Y, Tanabe A, Ono YJ, Hayashi M, Maeda K, Fujiwara S, Ashihara K, Nakamura M, Tanaka Y, et al: CD24 expression is a marker for predicting clinical outcome and regulates the epithelial-mesenchymal transition in ovarian cancer via both the Akt and ERK pathways. Oncol Rep 37: 3189-3200, 2017

20. Yoshida M, Takimoto R, Murase K, Sato Y, Hirakawa M, Tamura F, Sato T, Iyama S, Osuga T, Miyanishi K, et al: Targeting anticancer drug delivery to pancreatic cancer cells using a fucose-bound nanoparticle approach. PLoS One 7: e39545, 2012.

21. Hirai M, Minematsu H, Kondo N, Oie K, Igarashi K and Yamazaki N: Accumulation of liposome with Sialyl Lewis X to inflammation and tumor region: Application to in vivo bio-imaging. Biochem Biophys Res Commun 353: 553-558, 2007.

22. Hirai M, Hiramatsu Y, Iwashita S, Otani T, Chen L, Li YG, Okada M, Oie K, Igarashi K, Wakita $\mathrm{H}$ and Seno M: E-selectin targeting to visualize tumors in vivo. Contrast Media Mol Imaging 5: 70-77, 2010.

23. Pollmann D, Broekaert JA, Leis F, Tschopel P and Tolg G: Determination of boron in biological tissues by inductively-coupled plasma optical-emission spectrometry (ICP-OES) Fresen J Anal Chem 346: 441-445, 1993.

24. Duffy M and Thomas R: Benefits of a dual-view ICP-OES for the determination of boron, phosphorus, and sulfur in low alloy steels return to document menu. Atom Spectrosc 17: 128-132, 1996.

25. Lubura M, Hesse D, Neumann N, Scherneck S, Wiedmer P and Schürmann A: Non-invasive quantification of white and brown adipose tissues and liver fat content by computed tomography in mice. PLoS One 7: e37026, 2012.

26. Sano A, Kato H, Sakurai S, Sakai M, Tanaka N, Inose T, Saito K, Sohda M, Nakajima M, Nakajima T and Kuwano H: CD24 expression is a novel prognostic factor in esophageal squamous cell carcinoma. Ann Surg Oncol 16: 506-514, 2009.

27. Blechschmidt K, Sassen S, Schmalfeldt B, Schuster T, Höfler H and Becker KF: The E-cadherin repressor Snail is associated with lower overall survival of ovarian cancer patients. Br J Cancer 98: 489-495, 2008.

28. Kawaguchi H, Terai Y, Tanabe A, Sasaki H, Takai M, Fujiwara S, Ashihara K, Tanaka Y, Tanaka T, Tsunetoh S, et al: Gemcitabine as a molecular targeting agent that blocks the Akt cascade in platinum-resistant ovarian cancer. J Ovarian Res 7: 38, 2014.

29. Krantz SB, Shields MA, Dangi-Garimella S, Munshi HG and Bentrem DJ: Contribution of epithelial-to-mesenchymal transition and cancer stem cells to pancreatic cancer progression. J Surg Res 173: 105-112, 2012.

30. Peer D, Karp JM, Hong S, Farokhzad OC, Margalit R and Langer R: Nanocarriers as an emerging platform for cancer therapy. Nat Nanotechnol 2: 751-760, 2007.

31. Levi FA, Hrushesky WJ, Halberg F, Langevin TR, Haus E and Kennedy BJ: Lethal nephrotoxicity and hematologic toxicity of cis-diammine-dichloroplatinum ameliorated by optimal circadian timing and hydration. Eur J Cancer Clin Oncol 18: 471-477, 1982.

32. Peinado $\mathrm{H}$, Olmeda D and Cano A: Snail, Zeb and bHLH factors in tumour progression: An alliance against the epithelial phenotype? Nat Rev Cancer 7: 415-428, 2007.

33. Taki M, Abiko K, Baba T, Hamanishi J, Yamaguchi K, Murakami R, Yamanoi K, Horikawa N,Hosoe Y,Nakamura E, et al: Snail promotes ovarian cancer progression by recruiting myeloid-derived suppressor cells via CXCR2 ligand upregulation. Nat Commun 9: 1685, 2018.

This work is licensed under a Creative Commons Attribution-NonCommercial-NoDerivatives 4.0 International (CC BY-NC-ND 4.0) License. 